\title{
A cross-sectional assessment of health- related quality of life in Chinese patients with chronic hepatitis $c$ virus infection with EQ-5D
}

Rui Huang ${ }^{1}$, Huiying Rao ${ }^{1}$, Jia Shang ${ }^{2}$, Hong Chen ${ }^{3}$, Jun Li ${ }^{4}$, Qing Xie ${ }^{5}$, Zhiliang Gao ${ }^{6}$, Lei Wang ${ }^{7}$, Jia Wei ${ }^{8}$, Jianning Jiang ${ }^{9}$, Jian Sun ${ }^{10}$, Jiaji Jiang ${ }^{11}$ and Lai Wei ${ }^{1 *}$

\begin{abstract}
Background: Hepatitis C virus (HCV) infection is one of the most common liver infections, with a decrement in HRQOL of HCV patients. This study aims to assess Health-related quality of life (HRQoL) in Chinese patients with chronic HCV infection, and to identify significant predictors of the HRQOL in these patients of China.

Methods: In this cross-sectional observational study, treatment-naïve Han ethnic adults with chronic HCV infection were enrolled. Adopting European Quality of Life scale (EQ-5D) and EuroQOL visual analogue scale (EQ-VAS) were used to qualify HRQoL. Results were reported in descriptive analyses to describe sociodemographic and clinical characteristics. Multiple linear regression analysis was applied to investigate the associations of these variables with HRQoL. Binary logistic regression analysis was performed to identify associations of these variables with HRQoL by dimensions of EQ-5D.

Results: Nine hundred ninety-seven patients were enrolled in the study [median age $46.0(37.0,56.0)$ years; male 54.8\%]. Mean EQ-5D index and EQ-VAS score were $0.780 \pm 0.083$ and $77.2 \pm 14.8$. Multiple Linear regression analysis showed that income $(<2000 \mathrm{RMB}, \beta=-0.134 ; 2000-4999 \mathrm{RMB}, \beta=-0.085)$, moderate or severe symptoms of discomfort (more than one symptoms, $\beta=-0.090$ ), disease profile (cirrhosis, $\beta=-0.114$ ), hyperlipidemia $(\beta=-0.065)$ and depression $(\beta=-0.065)$ were independently associated with $E Q-5 D$ index. Residence (the west, $\beta=0.087)$, income $(<2000 R M B, \beta=-0.129 ; 2000-4999 R M B, \beta=-0.052)$, moderate or severe symptoms of discomfort (more than one symptoms, $\beta=-0.091$ ), disease profile and depression $(\beta=-0.316$ ) were the influencing factors on EQ-VAS. Binary logistic regression indicated that disease profile and clinical depression were the major influencing factors on all five dimensions of EQ-5D.
\end{abstract}

Conclusions: In this cross-sectional assessment of HCV patients in China, we indicated HRQoL of Chinese HCV patients. Significant negative associations between HRQoL and sociodemographic and clinical factors such as moderate or severe symptoms of discomfort, disease profile and depression emerged. We have to focus on optimally managing care of HCV patients and improving their HRQOL.

Trial registration: ClinicalTrials.gov identifier NCT01293279. Date of registration: February 10, 2011.

Keywords: Health related quality of life, Chronic hepatitis C, Euroqol EQ-5D, China

\footnotetext{
* Correspondence: weilai@pkuph.edu.cn

'Peking University People's Hospital, Peking University Hepatology Institute,

Beijing Key Laboratory for Hepatitis C and Immunotherapy for Liver Disease,

Beijing, China

Full list of author information is available at the end of the article
}

(c) The Author(s). 2018 Open Access This article is distributed under the terms of the Creative Commons Attribution 4.0 International License (http://creativecommons.org/licenses/by/4.0/), which permits unrestricted use, distribution, and reproduction in any medium, provided you give appropriate credit to the original author(s) and the source, provide a link to the Creative Commons license, and indicate if changes were made. The Creative Commons Public Domain Dedication waiver (http://creativecommons.org/publicdomain/zero/1.0/) applies to the data made available in this article, unless otherwise stated. 


\section{Background}

Hepatitis $\mathrm{C}$ virus (HCV) infection is one of the most common liver infections in the world. Total global anti-HCV prevalence is estimated at 2.5\% (177.5 million of HCV infected adults), ranging from $2.9 \%$ in Africa and $1.3 \%$ in Americas [1]. With chronic, life-long infections, hepatitis $\mathrm{C}$ virus causes liver cirrhosis and hepatocellular carcinoma (HCC) progressively. Globally, viral hepatitis accounted for 1.45 million deaths in 2013, half of which were caused by hepatitis $\mathrm{C}$ infections [2]. Chronic hepatitis $\mathrm{C}$ infection is a public health issue of utmost importance in the world, especially in China, which has 10 million patients suffering from this disease [3].

As the move from a disease-centered to a biopsychosocial model of medicine, health-related quality of life (HRQoL) is getting more and more concerns. It's especially important in management of chronic diseases, including chronic $\mathrm{HCV}$ infections. With growing interest in HRQoL these years, there have been many studies focusing on HRQoL of patients with chronic HCV infections. Compared to the general population, patients with $\mathrm{HCV}$ infection were reported to have a decrement in HRQoL $[4,5]$. It has been shown that the impairment in HRQoL for individuals with $\mathrm{HCV}$ was equivalent to, or even more severe than the impact on physical and general health experienced by patients with other common chronic conditions such as hypertension, diabetes or arthritis [6].

As known, the impact of disease on HRQoL may vary from population to population because of differences in socioeconomic status, cultural heritage, religion and life style. However, few studies have evaluated HRQoL of patients with HCV in China [7]. EQ-5D is a standardised instrument for use as a measure of health outcome (http:// www.euroqol.org/about-eq-5d.html), widely used in measurement of HRQoL in certain patients, and also in those with $\mathrm{HCV}$ infection $[8,9]$. Basing on the widely use of EQ-5D worldwide, we applied it in our study. The aim of this study was to assess HRQoL in Chinese patients with chronic $\mathrm{HCV}$ infection, to investigate whether variables related to sociodemographic characteristics, degree of liver impairment and clinical comorbidities are independently associated with HRQOL, and finally, to identify significant predictors of the HRQoL in these patients of China.

\section{Methods}

This study aims to assess HRQoL in Chinese patients with chronic $\mathrm{HCV}$ infection, and to identify significant predictors of the HRQoL in these patients of China.

\section{Study population}

This study was one part of the cross-sectional observational study, CCgenos (ClinicalTrials.gov identifier NCT01293279) [10]. To investigate the distribution of viral and host genotypes, only Han ethnic $\mathrm{HCV}$ treatment-naïve patients were enrolled. Those patients at least 18 years of age were seen at the outpatient department of 28 university-affiliated hospitals across China between February and June 2011. HCV infection was confirmed (anti-HCV antibody and HCV RNA positive) within 90 days prior to enrollment. Patients who had received antiviral or interferon-based therapy for hepatitis $C$ or hepatitis B were excluded; no other exclusion criteria were applied. Social-demographic and clinical information were collected within 9 days after enrollment, so was EQ-5D-3 L questionnaire. The respondents were asked to classify five dimensions of their own health status into three levels. They were asked to identify the point that they felt best illustrated their current overall health state on a visual analogue scale (VAS).

Social-demographic characteristics considered in this study included sex, age, residence (east, west, south, north, central), marital status (single, married, separated/ divorced/widowed), occupation (white collar, blue collar, unemployed, other), education attainment (primary junior, junior school, high school, college graduate) and economic status (monthly family income/per person < 2000 RMB, 2000-4999 RMB, $\geq 5000$ RMB) (1 RMB = 0.1457 USD.).

Clinical characteristics considered in this study included symptoms of discomfort (moderate or severe malaise, appetite decrease, nausea, discomfort of right hypochondrium, fever, xanthochromia, xanthurenic and other uncomfortable symptoms), disease profile (cirrhosis and chronic hepatitis, in which hepatitis without any moderate or severe uncomfortable symptoms mentioned above was considered asymptomatic, while the rest symptomatic), extrahepatic manifestations (Behcet's disease, cryoglobulinemia, prediabetes, diabetes, hyperlipidemia, fatty liver, insulin resistance, fibromyalgia, membranoproliferative glomerulonephritis, membranous nephropathy, multiple myeloma, Raynaud's syndrome, rheumatoid arthritis, Sjögren syndrome, systemic lupus erythematosus, thyroid dysfunction, skin lesions, hypertrophic cardiomyopathy, porphyria cutanea tarda, lichen planus, vasculitis, pulmonary fibrosis). In addition, comorbidities diagnosed by doctors including hepatitis $\mathrm{B}$, fatty liver, hyperlipidemia and diabetes were considered. Beck's depression score, which suggested clinical depression with score $\geq$ 17 was calculated.

Cirrhosis was diagnosed by liver biopsy or the presence of ascites, hepatic encephalopathy, upper gastrointestinal bleeding, or Child-Turcotte-Pugh score $>7$, or by any two of the following criteria: radiologic imaging showing nodular liver or splenomegaly, platelet count $<100 \times 10^{9} / \mathrm{L}$ in the absence of other explanations, liver stiffness $>13 \mathrm{kPA}$, or endoscopic detection of gastroesophageal varices. 


\section{Ethical approval}

The study was conducted in accordance with the International Society for Pharmacoepidemiology guidelines for Good Epidemiology Practices and applicable regulatory requirements. All patients provided written informed consent before participating.

\section{EQ-5D}

We used an approved Chinese version of the EQ-5D-3 L which has been tested for its validity and reliability in populations in mainland China [11, 12], EQ- 5D-3 L is the simplest multidimensional measure, composed of the EQ-5D descriptive system and the EQ visual analogue scale (EQ-VAS). The EQ-5D-3 L descriptive system classified patients' health status at three levels of severity (no, moderate, or severe problems) in five dimensions (mobility; self-care; usual activities; pain/ discomfort; anxiety/depression), resulting in scores that can be converted into a single index value for health status $(1=$ full health; $0=$ dead). Since no verified Chinese value set is available, the index value was assigned using a Japanese time trade-off value set [13], in which intercept was 0.152 ; mobility (none: 0.000 ; moderate: 0.075 ; severe: 0.418), self-care (none: 0.000; moderate: 0.054; severe: 0.102), usual activity (none: 0.000 ; moderate:0.044; severe: 0.133); pain/discomfort (none: 0.000; moderate: 0.080; severe: 0.194) and anxiety/depression (none: 0.000 ; moderate: 0.063; severe: 0.112) had correspondent values in three grade. In addition to the self-classifier, the EQ-5D contains a $20 \mathrm{~cm}$ visual analog scale (VAS) ranging from 0 (worst imaginable health) to 100 (best imaginable health) along which the respondent rates their health today. The index-based utility scores can be used to compare a burden of disease across different conditions. A single summary score was generated by applying societal preference weights to a health state classifier completed by patients.

\section{Statistical analysis}

Descriptive analysis of patients' demographic information was performed. Categorical variables were measured as percentages while continuous variables were expressed as mean \pm standard deviation. Mann-Whitney (gender, fatty liver, hyperlipidemia, diabetes, Beck's depression score) and Kruskal Wallis (age, residence, marital status, occupation, education, income, symptoms of discomfort, disease profile, and extrahepatic manifestation) tests were used as Kolmogrov-Smirnov test revealed nonnormal distribution of the data. A multiple linear regression analysis was applied to investigate the associations of social-demographic and clinical characteristics with HRQoL to select those linked to EQ index and EQ-VAS. A binary logistic regression analysis was performed to identify associations of these variables with HRQoL by dimensions of EQ-5D to select those linked to impairment in individual dimension of the EQ-5D instruments. A statistical value of $p<0.05$ was taken as significant. All analyses were performed using SPSS 23.0 (SPSS Inc., Chicago, IL).

\section{Results \\ Study population}

One thousand and twelve patients enrolled, in whom 997 patients met the protocol criteria for inclusion, while 15 were excluded for protocol violations.

\section{Patient sociodemographic and clinical status}

The sociodemographic characteristics of the patients were listed in Table 1. Patients had a median of 46 years of age and $546(54.8 \%)$ were males.

The clinical characteristics of the patients were listed in Table 2. 856 (86.1\%) patients didn't report any moderate or severe symptoms of discomfort, such as appetite decrease, nausea. Cirrhosis was reported in 101(10.1\%) patients. $41(4.1 \%)$ patients had a coinfection of hepatitis $\mathrm{B}$ and $\mathrm{C}$. Beck's depression score more than 17, suggesting clinical depression were seen in 168 (16.9\%) patients.

\section{Health-related quality of life}

Mean EQ-5D descriptive score and EQ-VAS were $0.780 \pm$ 0.083 and $77.2 \pm 14.8$, respectively. 79 (7.9\%), 24(2.4\%), and $71(7.1 \%)$ patients reported some problem (moderate or severe) in the mobility, self-care and usual activities respectively, whereas 343 (34.2\%) patients indicated some pain or discomfort (moderate or severe) in the fourth domain and 341 (34.1\%) reported moderate or severe anxiety or depression in the fifth domain as shown in Table 3.

\section{EQ-5D by sociodemographic and clinical characteristics}

EQ-5D index was significantly different by gender $(0.792 \pm 0.087$ vs $0.777 \pm 0.078, p=0.000)$, age $(p=$ $0.000)$, marital status $(p=0.000)$, education $(p=0.000)$, income $(p=0.001)$, symptoms of discomfort $(p=0.000)$, disease profile $(p=0.000)$, fatty liver $(0.772 \pm 0.100$ vs $0.788 \pm 0.083, p=0.041)$, hyperlipidemia $(0.740 \pm 0.110$ vs $0.785 \pm 0.087, p=0.008)$, diabetes $(0.767 \pm 0.101$ vs $0.786 \pm 0.086, p=0.037)$ and Beck's depression score $(0.798 \pm 0.074$ vs $0.712 \pm 0.115, p=0.000)$. EQ-VAS was significantly different by age $(p=0.000)$, residence $(p=$ $0.000)$, marital status $(p=0.006)$, income $(p=0.001)$, symptoms of discomfort $(p=0.000)$, disease profile $(p=$ $0.000)$, symptoms of discomfort $(p=0.000)$, Beck's depression score $(79.5 \pm 13.1$ vs $66.0 \pm 17.4, p=0.000)$. (Table 4 and Table 5).

\section{Multiple linear regression analyses on EQ-5D}

A Multiple Linear regression model was applied to investigate the effects of sociodemographic and clinical variables on HRQoL in patients with HCV (Table 6). 
Table 1 Sociodemographic characteristics of patients. (near Line 263)

\begin{tabular}{|c|c|}
\hline Parameter & \\
\hline \multicolumn{2}{|l|}{ Gender } \\
\hline Male, $n(\%)$ & $546(54.8)$ \\
\hline Female, $n(\%)$ & $451(45.2)$ \\
\hline Age, median years, (Q1, Q3) & $46.0(37.0,56.0)$ \\
\hline $18-39, n(\%)$ & $324(32.5)$ \\
\hline $40-59, n(\%)$ & $525(52.7)$ \\
\hline$\geq 60, n(\%)$ & $148(14.8)$ \\
\hline \multicolumn{2}{|l|}{ Residence } \\
\hline East, $n(\%)$ & $206(20.7)$ \\
\hline West, $n(\%)$ & $217(21.8)$ \\
\hline South, $n$ (\%) & $152(15.2)$ \\
\hline North, $n(\%)$ & $181(18.2)$ \\
\hline Central, $n(\%)$ & $241(24.2)$ \\
\hline \multicolumn{2}{|l|}{ Marital status } \\
\hline Single & $116(11.6)$ \\
\hline Married & $842(84.5)$ \\
\hline Separated/Divorced/Widowed & $39(3.9 \%)$ \\
\hline \multicolumn{2}{|l|}{ Occupation } \\
\hline White collar & $260(26.1)$ \\
\hline Blue collar & $414(41.5 \%)$ \\
\hline Unemployed & $179(18.0)$ \\
\hline Other & $144(14.4)$ \\
\hline \multicolumn{2}{|l|}{ Education } \\
\hline Primary School, $n$ (\%) & $177(17.7)$ \\
\hline Junior school, $n(\%)$ & $271(21.8)$ \\
\hline High-school, $n(\%)$ & $273(27.4)$ \\
\hline College graduate, $n$ (\%) & $276(27.6)$ \\
\hline \multicolumn{2}{|l|}{ Monthly family income/per person ${ }^{a}$} \\
\hline$<2000 \mathrm{RMB}$ & $558(56.0)$ \\
\hline 2000-4999 RMB & $352(35.3)$ \\
\hline$\geq 5000 \mathrm{RMB}$ & $87(8.7)$ \\
\hline
\end{tabular}

${ }^{\mathrm{a}} 1 \mathrm{RMB}=0.1457$ USD

EQ-5D index was negatively associated with symptoms of discomfort, disease progression, hyperlipidemia $(\beta=-$ $0.065)$ and Beck's depression score $(\beta=-0.294)$. Compared to patients without any moderate or severe symptoms, those had one $(\beta=-0.056)$ or more $(\beta=-0.090)$ symptoms reported lower EQ-5D index. When compared to patients with asymptomatic chronic hepatitis, those with any symptoms $(\beta=-0.078)$ or cirrhosis $(\beta=-0.114)$ indicated lower EQ-5D index. Income was positively associated with EQ-5D index $(<2000 \mathrm{RMB}, \beta=-0.134 ; 2000-4999$ $\mathrm{RMB}, \beta=-0.085)$.

EQ-VAS was negatively associated with symptoms of discomfort, disease progression and Beck's depression
Table 2 Clinical characteristics of patients. (near Line 273)

\section{Parameter}

Number of moderate or severe symptoms of discomfort, $n(\%)^{a}$

0

$858(86.1)$

1

$93(9.3)$

$\geq 2$

46(4.6)

Disease profile, $n(\%)$

Chronic hepatitis, asymptomatic ${ }^{b}$

$404(40.5)$

Chronic hepatitis, symptomatic

Cirrhosis

$101(10.1)$

Extrahepatic manifestations, $n(\%)^{c}$

Yes

166 (16.6)

No

$831(83.4)$

Hepatitis B

Yes

$41(4.1)$

No

$956(95.9)$

Fatty liver

Yes

$242(24.3)$

No

755 (75.7)

Hyperlipidemia

Yes

$22(2.2)$

No

$975(97.8)$

Diabetes

Yes

$81(8.1)$

No

$916(91.9)$

Beck's Depression Score, median (Q1, Q3)

$9.2(3.0,13.0)$

$<17$

$829(83.1$

$\geq 17$

$168(16.9)$

ancomfortable symptoms: moderate or above malaise, appetite decrease, nausea, discomfort of right hypochondrium, fever, xanthochromia, xanthurenic and other uncomfortable symptoms. ${ }^{b}$ Chronic hepatitis, asymptomatic: chronic hepatitis without any discomfortable complain above. 'Extrahepatic manifestation: Behcet's disease, cryoglobulinemia, prediabetes, diabetes, hyperlipidemia, fatty liver, insulin resistance, fibromyalgia, membranoproliferative glomerulonephritis, membranous nephropathy, multiple myeloma, Raynaud's syndrome, rheumatoid arthritis, Sjögren syndrome, systemic lupus erythematosus, thyroid dysfunction, skin lesions, hypertrophic cardiomyopathy, porphyria cutanea tarda, lichen planus, vasculitis, pulmonary fibrosis

Table 3 EQ-5D results of patients with hepatitis C. (near Line 304)

\begin{tabular}{llll}
\hline EQ-5D & None, $n(\%)$ & Moderate, $n(\%)$ & Severe, $n(\%)$ \\
\hline Mobility difficulties & $918(92.1)$ & $74(7.4)$ & $5(0.5)$ \\
Self-care difficulties & $973(97.6)$ & $22(2.2)$ & $2(0.2)$ \\
Usual activities difficulties & $926(92.9)$ & $68(6.8)$ & $3(0.3)$ \\
Pain/discomfort & $655(65.7)$ & $333(33.4)$ & $9(0.9)$ \\
Anxiety/depression & $656(65.8)$ & $313(31.4)$ & $28(2.8)$ \\
EQ-5D index & $0.780 \pm 0.083$ & \\
EQ-5D VAS & $0.780 \pm 0.083$ & \\
\hline
\end{tabular}


Table 4 Mean EQ-5D indexes and EQ-VAS scores by sociodemographic characteristics. (near Line 317)

\begin{tabular}{|c|c|c|c|c|c|c|}
\hline Parameter & Mean EQ-5D index & SD & $p$ value & Mean EQ-VAS score & SD & $p$ value \\
\hline \multicolumn{7}{|l|}{ Gender $^{\mathrm{b}}$} \\
\hline Males & 0.792 & 0.087 & 0.000 & 77.7 & 14.7 & 0.222 \\
\hline Females & 0.777 & 0.078 & & 76.7 & 14.8 & \\
\hline \multicolumn{7}{|l|}{$\mathrm{Age}^{\mathrm{a}}$} \\
\hline $18-39$ & 0.813 & 0.054 & 0.000 & 82.2 & 11.2 & 0.000 \\
\hline $40-59$ & 0.790 & 0.077 & & 78.6 & 13.8 & \\
\hline$\geq 60$ & 0.771 & 0.081 & & 75.4 & 14.1 & \\
\hline \multicolumn{7}{|l|}{ Residence $^{a}$} \\
\hline East & 0.781 & 0.091 & 0.115 & 74.4 & 12.8 & 0.000 \\
\hline West & 0.788 & 0.090 & & 79.9 & 15.2 & \\
\hline South & 0.789 & 0.073 & & 76.7 & 15.0 & \\
\hline North & 0.776 & 0.081 & & 77.6 & 15.6 & \\
\hline Central & 0.790 & 0.077 & & 77.3 & 14.9 & \\
\hline \multicolumn{7}{|l|}{ Marital status ${ }^{a}$} \\
\hline Single & 0.806 & 0.062 & 0.000 & 80.4 & 13.9 & 0.006 \\
\hline Married & 0.785 & 0.083 & & 77.1 & 14.8 & \\
\hline Separated/Divorced/Widowed & 0.730 & 0.114 & & 72.0 & 16.2 & \\
\hline \multicolumn{7}{|l|}{ Occupation $^{a}$} \\
\hline White collar & 0.792 & 0.080 & 0.054 & 78.6 & 14.1 & 0.173 \\
\hline Blue collar & 0.780 & 0080 & & 76.6 & 14.7 & \\
\hline Unemployed & 0.784 & 0.103 & & 77.7 & 15.1 & \\
\hline Other & 0.788 & 0.071 & & 76.0 & 14.8 & \\
\hline \multicolumn{7}{|l|}{ Education $^{a}$} \\
\hline Primary School & 0.763 & 0.102 & 0.000 & 75.7 & 16.1 & 0.105 \\
\hline Junior school & 0.782 & 0.100 & & 76.5 & 14.9 & \\
\hline High-school & 0.785 & 0.072 & & 77.1 & 14.8 & \\
\hline College graduate & 0.798 & 0.087 & & 79.1 & 13.6 & \\
\hline \multicolumn{7}{|l|}{ Monthly family income/per person ${ }^{a}$} \\
\hline$<2000 \mathrm{RMB}$ & 0.778 & 0.091 & 0.001 & 75.8 & 15.7 & 0.001 \\
\hline 2000-4999 RMB & 0.790 & 0.074 & & 78.5 & 13.7 & \\
\hline$\geq 5000 \mathrm{RMB}$ & 0.811 & 0.057 & & 81.5 & 11.5 & \\
\hline
\end{tabular}

${ }^{a}$ Kruskal Wallis Test. ${ }^{\mathrm{b}}$ Mann Whitney Test

score $(\beta=-0.316)$. Patients with one $(\beta=-0.046)$ or more $(\beta=-0.091)$ symptoms had lower EQ-VAS. Patients with any symptoms $(\beta=-0.107)$ or cirrhosis $(\beta=-0.099)$ reported lower EQ-VAS. Patients from the west of China had highest EQ-VAS, compared to those from other regions $(\beta=0.087)$. Income was positively associated with EQ-VAS $(<2000 \mathrm{RMB}, \beta=$ - 0.129; 2000-4999 RMB, $\beta=-0.052$ ).

\section{Logistic regression analyses in each EQ-5D dimension}

Further analyses comparing patients with and without any problem in each EQ-5D dimension by binary logistic regression indicated that disease profile and clinical depression were the major influencing factors on all the five dimensions (Table 7). Compared to asymptomatic chronic hepatitis, symptomatic chronic hepatitis and cirrhosis brought more problems in mobility, self-care, usual activities, pain/discomfort and anxiety/depression. Beck's depression score $\geq 17$ were also significantly associated with all five dimensions.

Old patients had more problems on the mobility dimension, and diabetes remained significantly associated with mobility $(\beta=0.696 ; p=0.049$; odds ratio $\mathrm{OR}=2.006 ; 95 \%$ CI 1.002-4.018). Problems on self-care were significantly associated with males $(\beta=-1.319 ; p=0.015$; OR $=0.267$; 95\% CI 0.093-0.771), residence, symptoms of discomfort and hyperlipidemia $(\beta=2.137 ; p=0.026$; $\mathrm{OR}=8.476 ; 95 \%$ CI 1.290-55.713): patients from the west had more 
Table 5 Mean EQ-5D indexes and EQ-VAS scores by clinical characteristics. (near Line 319)

\begin{tabular}{|c|c|c|c|c|c|c|}
\hline Parameter & EQ-5D index, mean & SD & $p$ value & EQ VAS, mean & SD & $p$ value \\
\hline \multicolumn{7}{|c|}{ Number of moderate or severe symptoms of discomfort ${ }^{a}$} \\
\hline 0 & 0.792 & 0.081 & 0.000 & 78.5 & 14.0 & 0.000 \\
\hline 1 & 0.746 & 0.093 & & 71.6 & 15.7 & \\
\hline$\geq 2$ & 0.719 & 0.087 & & 66.0 & 18.7 & \\
\hline \multicolumn{7}{|l|}{ Disease profile ${ }^{a}$} \\
\hline Chronic hepatitis, asymptomatic & 0.804 & 0.081 & 0.000 & 80.7 & 13.8 & 0.000 \\
\hline Chronic hepatitis, symptomatic & 0.774 & 0.076 & & 75.2 & 15.0 & \\
\hline Cirrhosis & 0.751 & 0.133 & & 73.3 & 13.8 & \\
\hline \multicolumn{7}{|l|}{ Extrahepatic manifestation ${ }^{a}$} \\
\hline Yes & 0.779 & 0.100 & 0.487 & 76.0 & 15.8 & 0.068 \\
\hline No & 0.787 & 0.086 & & 77.7 & 14.5 & \\
\hline \multicolumn{7}{|l|}{ Hepatitis B ${ }^{b}$} \\
\hline Yes & 0.771 & 0.115 & 0.791 & 72.5 & 16.0 & 0.051 \\
\hline No & 0.785 & 0.086 & & 77.4 & 14.7 & \\
\hline \multicolumn{7}{|l|}{ Fatty liver ${ }^{b}$} \\
\hline Yes & 0.772 & 0.100 & 0.041 & 76.4 & 15.3 & 0.552 \\
\hline No & 0.788 & 0.083 & & 77.5 & 14.6 & \\
\hline \multicolumn{7}{|l|}{ Hyperlipidemia $^{\mathrm{b}}$} \\
\hline Yes & 0.740 & 0.110 & 0.008 & 72.5 & 13.0 & 0.052 \\
\hline No & 0.785 & 0.087 & & 77.3 & 14.8 & \\
\hline \multicolumn{7}{|l|}{ Diabetes $^{b}$} \\
\hline Yes & 0.767 & 0.101 & 0.037 & 74.0 & 15.9 & 0.053 \\
\hline No & 0.786 & 0.086 & & 77.5 & 15.9 & \\
\hline \multicolumn{7}{|l|}{ Beck's Depression Score ${ }^{b}$} \\
\hline$<17$ & 0.798 & 0.074 & 0.000 & 79.5 & 13.1 & 0.000 \\
\hline$\geq 17$ & 0.712 & 0.115 & & 66.0 & 17.4 & \\
\hline
\end{tabular}

${ }^{\mathrm{a}}$ Kruskal Wallis Test. ${ }^{\mathrm{b}}$ Mann Whitney Test

problem of self-care than those from other regions $(\beta$ $=0.265 ; p=0.688 ; \mathrm{OR}=1.303 ; 95 \%$ CI $0.357-4.752)$; patients with moderate or severe symptoms of discomforts had seven times more likely to have problem in self-care $(\beta=1.960 ; p=0.002$; $\mathrm{OR}=7.103 ; 95 \%$ CI 2.043-24.692). More problems in usual activities were significantly associated with $\geq 2$ symptoms of discomforts $(\beta=1.159 ; \quad p=0.008 ; \quad \mathrm{OR}=3.186 ; 95 \% \mathrm{CI}$ $1.355-7.495)$. Females $(\beta=0.057 ; p=0.000 ; \quad \mathrm{OR}=$ $2.748 ; 95 \%$ CI $2.748-2.375)$, the old age $(\beta=1.011 ; p$ $=0.009 ; \mathrm{OR}=1.746 ; 95 \%$ CI $1.285-5.876)$ and hyperlipidemia $(\beta=1.092 ; \quad p=0.033 ; \quad \mathrm{OR}=2.981 ; 95 \% \mathrm{CI}$ $1.090-8.150)$ remained significantly associated with pain/discomfort. Besides, more pain/discomfort were significantly associated with $\geq 2$ symptoms of discomforts $(\beta=0.573 ; p=0.032$; OR $=1.774 ; 95 \%$ CI $1.051-$ 2.993). Females $(\beta=0.322 ; p=0.041 ; \mathrm{OR}=1.380 ; 95 \%$ CI 1.013-1.879) and low income $(\beta=0.628 ; p=0.041$; $\mathrm{OR}=1.874 ; 95 \%$ CI $1.025-3.424$ ) were significantly associated with anxiety/depression.

\section{Discussion}

With 118.9 million HCV RNA positive cases worldwide [1], it is important to understand the effects of HCV infection on patients' well-being and HRQoL, by assessing the health outcome reported by these patients. It would help to improve their care management. Poor HRQoL were demonstrated in these Chinese patients with chronic HCV infection. Worsening HRQoL was significantly associated with unstable marital status, residence, poor family income, more symptoms of moderate or severe discomfort, disease progression of hepatitis $\mathrm{C}$ and some comorbidities such as hyperlipidemia and depression. This is a multi-centric, large-sample assessment of HRQoL in Chinese patients with HCV. Although HRQoL of patients with $\mathrm{HCV}$ has been evaluated in several counties and regions, the current study from mainland China may provide important evidence for expanding the knowledge about the impact of chronic hepatitis $\mathrm{C}$ infection on health of people. 
Table 6 Multiple linear regression analyses on EQ-5D index score and EQ-VAS scores. (near Line 344)

\begin{tabular}{|c|c|c|c|c|c|c|c|c|c|c|}
\hline \multirow[t]{3}{*}{ Parameter } & \multicolumn{6}{|l|}{ EQ-5D index } & \multicolumn{4}{|c|}{ EQ-VAS score } \\
\hline & \multirow[t]{2}{*}{$\mathrm{B}^{\mathrm{a}} \pm \mathrm{SE}$} & \multirow{2}{*}{$\begin{array}{l}\beta \\
\text { estimate }^{b}\end{array}$} & \multirow{2}{*}{$\begin{array}{l}p \\
\text { value }\end{array}$} & \multicolumn{2}{|c|}{$95.0 \% \mathrm{Cl}$ for B } & \multirow[t]{2}{*}{$B^{a} \pm S E$} & \multirow{2}{*}{$\begin{array}{l}\beta \\
\text { estimate }^{b}\end{array}$} & \multirow{2}{*}{$\begin{array}{l}p \\
\text { value }\end{array}$} & \multirow[t]{2}{*}{$95.0 \% \mathrm{Cl}$} & \\
\hline & & & & Lower & Upper & & & & & \\
\hline Intercept & $0.839 \pm 0.018$ & & 0.000 & 0.803 & 0.875 & $82.238 \pm 3.112$ & & 0.000 & 76.132 & 88.344 \\
\hline Gender (Ref: Male) & $-0.007 \pm 0.005$ & -0.042 & 0.176 & -0.018 & 0.003 & $0.207 \pm 0.920$ & 0.007 & 0.822 & -1.592 & 2.012 \\
\hline \multicolumn{11}{|l|}{ Age (Ref: $\geq 60 y$ ) } \\
\hline $18-29$ & $0.016 \pm 0.012$ & 0.062 & 0.198 & -0.008 & 0.040 & $3.991 \pm 2.101$ & 0.091 & 0.058 & -0.132 & 8.113 \\
\hline $30-39$ & $0.007 \pm 0.009$ & 0.030 & 0.477 & -0.012 & 0.025 & $0.968 \pm 1.587$ & 0.026 & 0.542 & -2.147 & 4.083 \\
\hline $40-49$ & $0.011 \pm 0.008$ & 0.055 & 0.203 & -0.006 & 0.027 & $2.372 \pm 1.433$ & 0.072 & 0.098 & -0.440 & 5.184 \\
\hline $50-59$ & $0.007 \pm 0.008$ & 0.035 & 0.400 & -0.009 & 0.023 & $0.799 \pm 1.411$ & 0.023 & 0.571 & -1.970 & 3.569 \\
\hline \multicolumn{11}{|l|}{ Residence (Ref: Central) } \\
\hline East & $-0.002 \pm 0.008$ & -0.011 & 0.768 & -0.018 & 0.013 & $-2.047 \pm 1.338$ & -0.056 & 0.126 & -4.672 & 0.579 \\
\hline West & $0.002 \pm 0.008$ & -0.010 & 0.777 & -0.013 & 0.017 & $3.115 \pm 1.280$ & 0.087 & 0.015 & 0.603 & 5.627 \\
\hline South & $-0.001 \pm 0.009$ & -0.006 & 0.873 & -0.018 & 0.015 & $-1.004 \pm 1.445$ & -0.024 & 0.487 & -3.841 & 1.832 \\
\hline North & $-0.005 \pm 0.009$ & -0.021 & 0.575 & -0.022 & 0.012 & $1.467 \pm 1.438$ & 0.038 & 0.308 & -1.355 & 4.289 \\
\hline \multicolumn{11}{|l|}{ Marital status (Ref: Single) } \\
\hline Married & $-0.007 \pm 0.011$ & -0.030 & 0.500 & -0.029 & 0.014 & $-0.741 \pm 1.833$ & -0.018 & 0.686 & -4.339 & 2.857 \\
\hline Separated/Divorced/Widowed & $-0.033 \pm 0.017$ & -0.073 & 0.052 & -0.066 & -0.000 & $-0.671 \pm 2.856$ & -0.009 & 0.814 & -6.277 & 4.934 \\
\hline \multicolumn{11}{|l|}{ Education (Ref: Primary school) } \\
\hline Junior school & $-0.004 \pm 0.009$ & -0.018 & 0.631 & -0.021 & 0.013 & $2.410 \pm 1.481$ & 0.0062 & 0.104 & -0.496 & 5.315 \\
\hline High-school & $0.005 \pm 0.007$ & 0.023 & 0.538 & -0.010 & 0.019 & $1.640 \pm 1.256$ & 0.049 & 0.192 & -0.825 & 4.104 \\
\hline College graduate & $0.002 \pm 0.007$ & 0.010 & 0.790 & -0.012 & 0.016 & $0.749 \pm 1.192$ & 0.023 & 0.530 & -1.590 & 3.087 \\
\hline \multicolumn{11}{|c|}{ Monthly family income/per person ( $\geq 5000$ RMB) } \\
\hline$<2000$ RMB & $-0.024 \pm 0.010$ & -0.134 & 0.017 & -0.043 & -0.004 & $-3.833 \pm 1.669$ & -0.129 & 0.022 & -7.107 & -0.558 \\
\hline 2000-4999 RMB & $-0.016 \pm 0.010$ & -0.085 & 0.110 & -0.035 & 0.004 & $-1.612 \pm 1.647$ & -0.052 & 0.328 & -4.844 & 1.621 \\
\hline \multicolumn{11}{|c|}{ Number of moderate or severe symptoms of discomfort (Ref:0) } \\
\hline 1 & $-0.017 \pm 0.009$ & -0.056 & 0.074 & -0.035 & 0.002 & $-2.358 \pm 1.588$ & -0.046 & 0.138 & -5.474 & 0.758 \\
\hline$\geq 2$ & $-0.038 \pm 0013$ & -0.090 & 0.003 & -0.062 & -0.013 & $-6.410 \pm 2.124$ & -0.091 & 0.003 & -10.578 & -2.241 \\
\hline \multicolumn{11}{|c|}{ Disease profile (Ref: Chronic hepatitis, asymptomatic) } \\
\hline Chronic hepatitis, symptomatic & $-0.014 \pm 0.006$ & -0.078 & 0.022 & -0.025 & -0.002 & $-3.167 \pm 1.003$ & -0.107 & 0.002 & -5.135 & -1.199 \\
\hline cirrhosis & $-0.033 \pm 0.010$ & -0.114 & 0.001 & -0.052 & -0.014 & $-4.834 \pm 1.630$ & -0.099 & 0.003 & -8.032 & -1.636 \\
\hline Fatty liver (Ref: No) & $0.003 \pm 0.009$ & 0.011 & 0.710 & -0.014 & 0.021 & $-0.629 \pm 1.524$ & -0.013 & 0.680 & -3.621 & 2.362 \\
\hline Hyperlipidemia (Ref: No) & $-0.039 \pm 0.018$ & -0.065 & 0.031 & -0.074 & -0.003 & $-3.147 \pm 3.046$ & -0.031 & 0.302 & -9.125 & 2.831 \\
\hline Diabetes (Ref: No) & $-0.011 \pm 0.010$ & -0.033 & 0.272 & -0.030 & 0.008 & $-2.616 \pm 1.632$ & -0.048 & 0.109 & -5.819 & 0.586 \\
\hline Beck's Depression Score (Ref: < 17) & $-0.069 \pm 0.007$ & -0.294 & 0.000 & -0.083 & -0.054 & $-12.453 \pm 1.220$ & -0.316 & 0.000 & -14.848 & -10.059 \\
\hline
\end{tabular}

${ }^{a}$ Unstandardized sample regression coefficient

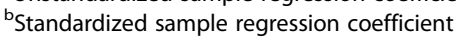

Although majority of $\mathrm{HCV}$-infected patients are asymptomatic during its natural course, $\mathrm{HCV}$ itself may still decrease the HRQOL no matter how advanced disease progression they have [14]. Studies in European and American countries indicated that $\mathrm{HCV}$ patients had a clinically meaningful decrement in quality of life compared to controls $[15,16]$. The relevant data from Asia was limited $[17,18]$, however, our result, that the mean EQ-5D index and EQ-VAS of patients with HCV infection in this study were $0.780 \pm 0.083$ and $77.2 \pm 14.8$ respectively brought the knowledge about the impact of $\mathrm{HCV}$ infection on Chinese patients' HRQoL.

Sociodemographic characteristics such as residence in the west of China, stable marital status, high family income have been found to be favorable to better quality of life for patients with $\mathrm{HCV}$ infection. Although the association between gender and HRQoL was not significant in our study, women still reported more problems in dimensions of pain/discomfort $(\mathrm{OR}=1.746 ; 95 \% \mathrm{CI}$ $1.283-2.375)$ and anxiety/depression $(\mathrm{OR}=1.380 ; 95 \%$ 
Table 7 Binary logistic regression analyses on having problem in each EQ-5D dimension. (near Line 359)

\begin{tabular}{|c|c|c|c|c|c|}
\hline \multirow[t]{2}{*}{ Independent variables } & \multirow[t]{2}{*}{$\mathrm{B}$} & \multirow[t]{2}{*}{$p$} & \multirow[t]{2}{*}{ Odds ratio } & \multicolumn{2}{|l|}{$95 \% \mathrm{Cl}$} \\
\hline & & & & Lower & Upper \\
\hline \multicolumn{6}{|l|}{ a. Dependent variable: mobility } \\
\hline \multicolumn{6}{|l|}{ Age (Ref: 17-29y) } \\
\hline $30-39$ & 1.526 & 0.183 & 4.598 & 0.488 & 43.360 \\
\hline $40-49$ & 1.318 & 0.257 & 3.736 & 0.383 & 36.450 \\
\hline $50-59$ & 1.938 & 0.094 & 6.948 & 0.719 & 67.187 \\
\hline$\geq 60$ & 2.819 & 0.015 & 16.759 & 1.733 & 162.099 \\
\hline \multicolumn{6}{|c|}{ Disease profile (Ref: Chronic hepatitis, asymptomatic) } \\
\hline Chronic hepatitis, symptomatic & 0.072 & 0.833 & 1.074 & 0.551 & 2.093 \\
\hline cirrhosis & 1.201 & 0.002 & 3.323 & 1.539 & 7.174 \\
\hline Diabetes (Ref: No) & 0.696 & 0.049 & 2.006 & 1.002 & 4.018 \\
\hline Beck's Depression Score, mean (Ref: < 17) & 0.881 & 0.004 & 2.414 & 1.326 & 4.396 \\
\hline \multicolumn{6}{|l|}{ b. Dependent variable: self-care } \\
\hline Gender (Ref: Male) & -1.319 & 0.015 & 0.267 & 0.093 & 0.771 \\
\hline \multicolumn{6}{|l|}{ Residence (Ref: Central) } \\
\hline East & 0.098 & 0.085 & 1.103 & 0.292 & 4.160 \\
\hline West & 0.265 & 0.688 & 1.303 & 0.357 & 4.752 \\
\hline South & -0.712 & 0.425 & 0.491 & 0.085 & 2.819 \\
\hline North & -2.111 & 0.043 & 0.121 & 0.016 & 0.939 \\
\hline \multicolumn{6}{|c|}{ Number of moderate or severe symptoms of discomfort (Ref:0) } \\
\hline 1 & 1.960 & 0.002 & 7.103 & 2.043 & 24.692 \\
\hline$\geq 2$ & 0.923 & 0.260 & 2.517 & 0.505 & 12.560 \\
\hline \multicolumn{6}{|c|}{ Disease profile (Ref: Chronic hepatitis, asymptomatic) } \\
\hline Chronic hepatitis, symptomatic & -1.170 & 0.105 & 0.310 & 0.075 & 1.278 \\
\hline cirrhosis & 1.333 & 0.046 & 3.793 & 1.023 & 14.062 \\
\hline Hyperlipidemia (Ref: No) & 2.137 & 0.026 & 8.476 & 1.290 & 55.713 \\
\hline Beck's Depression Score, mean (Ref: < 17) & 1.256 & 0.015 & 3.512 & 1.275 & 9.672 \\
\hline \multicolumn{6}{|l|}{ c. Dependent variable: usual activities } \\
\hline \multicolumn{6}{|c|}{ Number of moderate or severe symptoms of discomfort (Ref:0) } \\
\hline 1 & 0.707 & 0.061 & 2.028 & 0.968 & 4.253 \\
\hline$\geq 2$ & 1.159 & 0.008 & 3.186 & 1.355 & 7.495 \\
\hline \multicolumn{6}{|c|}{ Disease profile (Ref: Chronic hepatitis, asymptomatic) } \\
\hline Chronic hepatitis, symptomatic & 0.409 & 0.273 & 1.505 & 0.725 & 3.123 \\
\hline cirrhosis & 1.596 & 0.000 & 4.932 & 2.138 & 11.374 \\
\hline Beck's Depression Score, mean (Ref: < 17) & 0.796 & 0.008 & 2.218 & 1.230 & 3.998 \\
\hline \multicolumn{6}{|l|}{ d. Dependent variable: pain/discomfort } \\
\hline Gender (Ref: Male) & 0.057 & 0.000 & 1.746 & 1.283 & 2.375 \\
\hline \multicolumn{6}{|l|}{ Age (Ref: 17-29y) } \\
\hline $30-39$ & 0.616 & 0.088 & 1.851 & 0.913 & 3.753 \\
\hline $40-49$ & 0.621 & 0.082 & 1.860 & 0.925 & 3.742 \\
\hline $50-59$ & 0.735 & 0.047 & 2.086 & 1.008 & 4.317 \\
\hline$\geq 60$ & 1.011 & 0.009 & 2.748 & 1.285 & 5.876 \\
\hline \multicolumn{6}{|c|}{ Number of moderate or severe symptoms of discomfort (Ref:0) } \\
\hline 1 & 0.388 & 0.128 & 1.474 & 0.894 & 2.428 \\
\hline
\end{tabular}


Table 7 Binary logistic regression analyses on having problem in each EQ-5D dimension. (near Line 359) (Continued)

\begin{tabular}{|c|c|c|c|c|c|}
\hline \multirow[t]{2}{*}{ Independent variables } & \multirow[t]{2}{*}{ B } & \multirow[t]{2}{*}{$p$} & \multirow[t]{2}{*}{ Odds ratio } & \multicolumn{2}{|l|}{$95 \% \mathrm{Cl}$} \\
\hline & & & & Lower & Upper \\
\hline$\geq 2$ & 0.573 & 0.032 & 1.774 & 1.051 & 2.993 \\
\hline \multicolumn{6}{|c|}{ Disease profile (Ref: Chronic hepatitis, asymptomatic) } \\
\hline Chronic hepatitis, symptomatic & 0.767 & 0.000 & 2.154 & 1.527 & 3.038 \\
\hline cirrhosis & 0.573 & 0.032 & 1.774 & 1.051 & 2.993 \\
\hline Hyperlipidemia (Ref: No) & 1.092 & 0.033 & 2.981 & 1.090 & 8.150 \\
\hline Beck's Depression Score, mean (Ref: < 17) & 1.165 & 0.000 & 3.207 & 2.175 & 4.728 \\
\hline \multicolumn{6}{|l|}{ e. Dependent variable: anxiety/depression } \\
\hline Gender (Ref: Male) & 0.322 & 0.041 & 1.380 & 1.013 & 1.879 \\
\hline \multicolumn{6}{|c|}{ Monthly family income/per person ( $\geq 5000$ RMB) } \\
\hline$<2000$ RMB & 0.628 & 0.041 & 1.874 & 1.025 & 3.424 \\
\hline 2000-4999 RMB & 0.328 & 0.287 & 1.388 & 0.759 & 2.537 \\
\hline \multicolumn{6}{|c|}{ Disease profile (Ref: Chronic hepatitis, asymptomatic) } \\
\hline Chronic hepatitis, symptomatic & 0.570 & 0.001 & 1.769 & 1.263 & 2.476 \\
\hline cirrhosis & -0.310 & 0.302 & 0.733 & 0.407 & 1.322 \\
\hline Beck's Depression Score, mean (Ref: <17) & 1.891 & 0.000 & 6.623 & 4.428 & 9.907 \\
\hline
\end{tabular}

CI 1.013-1.879), which was in line with EQ-5D population health studies in both other countries and China [19-21] .Female patients with chronic hepatitis $C$ tended to be fatigued and depressive [22], which might be the reason why they had worse HRQoL than males. What's interesting we found was that patients from western China, who resided in less developed area of China, had significant higher EQ-VAS scores but more problems in self-care dimension, indicating that they considered themselves had relatively better health status, while their EQ-5D index didn't show the same superiority. The reason may be that patients from western China hadn't truly recognized their well-being and health status with less knowledge and education for hepatitis $C$ than those from developed areas. We should make further efforts on this issue to tell the reason why discrepancy existed by different areas across China in the future.

Clinical characteristics such as more symptoms of moderate or severe discomfort, advanced disease progression, hyperlipidemia and Beck's depression score were indicated to be associated with worse HRQoL. Patients with hyperlipidemia reported worse health status by EQ-5D index. As known, HCV infection induced hepatic steatosis and altered lipid metabolism [23]. Our results demonstrated the similar association: suffering from hyperlipidemia made EQ-5D Index significantly lower $(\beta=-0.065)$ by impacting on dimensions of self-care $(\mathrm{OR}=8.476 ; 95 \% \mathrm{CI}$ 1.290-55.713) and pain/discomfort $(\mathrm{OR}=2.981 ; 95 \% \mathrm{CI}$ 1.090-8.150). Limited literatures focused on the impact of hyperlipidemia on the quality of life among patients with $\mathrm{HCV}$ infection, and the only two relevant studies didn't show the association between hyperlipidemia and HRQoL in HCV population $[24,25]$, which we found in this study highlighted the potential correlation of hyperlipidemia and HRQoL in patients with chronic HCV infection.

Coinfection of HBV/HCV is seen in a substantial number of chronic liver disease cases, who have a higher risk of progression to cirrhosis and decompensated liver disease and HCC [26], especially in the Asian-Pacific region, with the reported prevalence of $\mathrm{HBV} / \mathrm{HCV}$ infection at $10-16 \%$ [27]. 41 (4.1\%) cases of HBV/HCV infection were included in our study: both EQ-5D index $(0.785 \pm 0.086$ vs $0.771 \pm 0.115, p=0.791)$ and EQ-VAS $(77.4 \pm 14.7$ vs 72.5 $\pm 16.0, p=0.051)$ of patients without hepatitis B were higher than those of $\mathrm{HBV} / \mathrm{HCV}$ patients. Cause no study has evaluated how $\mathrm{HBV} / \mathrm{HCV}$ coinfection impact on the HRQoL except one focused on the impact of hepatitis B and $\mathrm{C}$ coinfection on health-related quality of life in HIV positive individuals [28], our results illuminated our understanding of this issue.

The impact of fatigue on quality of life of HCV-infected patients has long been a focused issue [29,30]. However, the effect of other symptoms, such as malaise, appetite decrease, nausea, discomfort of right hypochondrium, on the HRQoL in patients with HCV has been overlooked. Questions were designed to assess uncomfortable symptoms, including malaise, appetite decrease, nausea, discomfort of right hypochondrium, fever, xanthochromia, xanthurenic and others, graded into mild, moderate and severe. Compared to no moderate or severe symptoms of discomfort, one or more symptoms were significant associated with worse EQ-5D index and EQ-VAS. Besides, more symptoms also brought more problems in dimensions of self-care, usual activity and pain/discomfort. 
- As was well-known, impairment in HRQoL in patients with $\mathrm{HCV}$ was associated with the severity of liver disease, patients with decompensated cirrhosis, exhibiting the highest impairment in HRQoL $[8,31]$. However, the number of subjects in end-stage liver disease (only 54 patients with decompensated cirrhosis) was small in 101 (10.1\%) patients with cirrhosis of our study, compared with the vast majority of patients having $\mathrm{HCV}$ in the absence of cirrhosis. Nevertheless, cirrhosis were found to be significantly associated with lower EQ-5D index $(\beta=-0.114)$ and EQ-VAS $(\beta=-0.099)$ by impacting on all five dimensions. Very few former studies of HRQoL in HCV have been undertaken in patients with HCV-induced decompensated cirrhosis. That may be our concern in the future.

The limitation of this study was that it was a cross-sectional study without health population as control. Cause the patients we included were treatment naïve, we can't tell the impact of antiviral therapy on HRQoL. We used a Japanese time trade-off value set to convert scores, from five dimensions (mobility; self-care; usual activities; pain/ discomfort; anxiety/depression), into a single index value for health status [13]. In spite of the widely usage of this Japanese time trade-off value set in Chinese population [32], it was still a limitation for our study. Future research is needed to validate the association between sociodemographic, clinical characteristics and HRQoL of patients in different liver disease stages of HCV. As the study of CCgenos is on-going, longitudinal assessment of HRQoL and influence of treatment on HRQoL will be performed.

\section{Conclusion}

In this cross-sectional assessment of patients with chronic HCV infection in China, we have shown that HRQoL in Chinese HCV patients impaired. Significant association between HRQoL and sociodemographic and clinical factors such as residence, income, moderate or severe symptoms of discomfort, disease profile, hyperlipidemia and depression. However, further study of these association is needed. We have to focus on optimally managing care of patients with chronic HCV infection and improving their HRQoL.

\section{Abbreviations}

ALD: Alcoholic liver disease; EQ-VAS: EQ visual analogue scale; HCC: Hepatocellular carcinoma; HCV: Hepatitis C virus; HRQoL: Health-related quality of life; NAFLD: Non-alcoholic fatty liver disease

\section{Acknowledgements}

This work was supported by grants from the China National Science and Technology Major Project for Infectious Diseases Control during the 11th Five-Year Plan Period [grant numbers 2008ZX10002-013, 2008ZX10002-012] and 12th Five-Year Plan Period [grant number 2012ZX10002003], and from Bristol-Myers Squibb.

\section{Funding}

This work was supported by grants from the China National Science and Technology Major Project for Infectious Diseases Control during the 11th Five-Year Plan Period [grant numbers 2008ZX10002-013, 2008ZX10002-012] and 12th Five-Year Plan Period [grant number 2012ZX10002003], and from Bristol-Myers Squibb.

\section{Availability of data and materials}

The datasets used and analysed during the current study are available from the corresponding author on reasonable request.

\section{Authors' contributions}

$\mathrm{RH}$ analyzed and interpreted the patient data regarding quality of life and was a major contributor in writing the manuscript. All authors read and approved the final manuscript.

\section{Ethics approval and consent to participate}

This study was approved by Medical ethics committee of Peking University People's Hospital. All patients provided written informed consent before participating.

\section{Competing interests}

$L$ Wei has received research support and/or consulting fees from BristolMyers Squibb, Roche, and Novartis. J Sun has received research support from Roche and Novartis. R Huang, H Rao, J Shang, H Chen, J Li, Q Xie, Z Gao, L Wang, J Wei, J Jiang and Y Sun have no conflicts.

\section{Publisher's Note}

Springer Nature remains neutral with regard to jurisdictional claims in published maps and institutional affiliations.

\section{Author details \\ 'Peking University People's Hospital, Peking University Hepatology Institute, Beijing Key Laboratory for Hepatitis C and Immunotherapy for Liver Disease, Beijing, China. ${ }^{2}$ Henan Provincial People's Hospital, Zhengzhou, China. ${ }^{3}$ First Hospital of Lanzhou University, Lanzhou, China. ${ }^{4}$ First Affiliated Hospital with Nanjing Medical University, Nanjing, China. ${ }^{5}$ Shanghai Ruijin Hospital, Shanghai, China. ${ }^{6}$ Third Affiliated Hospital of Sun Yat-sen University, Guangzhou, China. ${ }^{7}$ Second Hospital of Shandong University, Jinan, China. ${ }^{8}$ First Affiliated Hospital of Kunming Medical College, Kunming, China. ${ }^{9}$ First Affiliated Hospital of Guangxi Medical University, Nanning, China. ${ }^{10}$ Nanfang Hospital, Southern Medical University, Guangzhou, China. ${ }^{11}$ First Affiliated Hospital of Fujian Medical University, Fuzhou, China.}

Received: 24 November 2017 Accepted: 21 May 2018

Published online: 15 June 2018

References

1. Petruzziello A, Marigliano S, Loquercio G, Cozzolino A, Cacciapuoti C. Global epidemiology of hepatitis $C$ virus infection: an up-date of the distribution and circulation of hepatitis C virus genotypes. World J Gastroenterol. 2016; 22(34):7824-40.

2. Stanaway JD, Flaxman AD, Naghavi M, Fitzmaurice C, Vos T, Abubakar I, et al. The global burden of viral hepatitis from 1990 to 2013: findings from the global burden of disease study 2013. Lancet (London, England). 2016; 388(10049):1081-8.

3. Chen YS, Li L, Cui FQ, Xing WG, Wang L, Jia ZY, et al. A sero-epidemiological study on hepatitis $C$ in China. Zhonghua liu xing bing xue za zhi = Zhonghua liuxingbingxue zazhi. 2011;32(9):888-91.

4. Younossi Z, Park H, Henry L, Adeyemi A, Stepanova M. Extrahepatic manifestations of hepatitis C: a meta-analysis of prevalence, quality of life, and economic burden. Gastroenterology. 2016;150(7):1599-608.

5. Samp JC, Perry R, Piercy J, Wood R, Baran RW. Patient health utility, work productivity, and lifestyle impairment in chronic hepatitis $C$ patients in France. Clin Res Hepatol Gastroenterol. 2015;39(3):307-14.

6. Kallman J, O'Neil MM, Larive B, Boparai N, Calabrese L, Younossi ZM. Fatigue and health-related quality of life (HRQL) in chronic hepatitis $C$ virus infection. Dig Dis Sci. 2007;52(10):2531-9.

7. Ren H, Shi Y, Meng W, Hu JY, Chen YH, Pan QC. Study of disease burden of chronic hepatitis $B$ and $C$ patients in shanghai based on Bronfenbrenner' $s$ 
ecological systems theory: a community-based survey. Zhonghua liu xing bing xue za zhi = Zhonghua liuxingbingxue zazhi. 2017;38(1):37-42.

8. Bjornsson E, Verbaan H, Oksanen A, Fryden A, Johansson J, Friberg S, et al. Health-related quality of life in patients with different stages of liver disease induced by hepatitis C. Scand J Gastroenterol 2009;44(7):878-887.

9. Vargas CL, Espinoza MA, Giglio A, Soza A. Cost effectiveness of Daclatasvir/ Asunaprevir versus Peginterferon/ribavirin and protease inhibitors for the treatment of hepatitis c genotype 1b naive patients in Chile. PLoS One. 2015; 10(11):e0141660.

10. Rao H, Wei L, Lopez-Talavera JC, Shang J, Chen H, Li J, et al. Distribution and clinical correlates of viral and host genotypes in Chinese patients with chronic hepatitis C virus infection. J Gastroenterol Hepatol. 2014;29(3):545-53.

11. Wang H, Kindig DA, Mullahy J. Variation in Chinese population health related quality of life: results from a EuroQol study in Beijing. China Qual Life Res. 2005;14(1):119-32.

12. Wang HM, Patrick DL, Edwards TC, Skalicky AM, Zeng HY, Gu WW. Validation of the EQ-5D in a general population sample in urban China. Qual Life Res. 2012;21(1):155-60.

13. Tsuchiya A, lkeda S, lkegami N, Nishimura S, Sakai I, Fukuda T, et al. Estimating an EQ-5D population value set: the case of Japan. Health Econ. 2002;11(4):341-53.

14. Strauss E, Porto-Ferreira FA, de Almeida-Neto C, Teixeira MC. Altered quality of life in the early stages of chronic hepatitis $C$ is due to the virus itself. Clin Res Hepatol Gastroenterol. 2014;38(1):40-5.

15. Vietri J, Prajapati G, El Khoury AC. The burden of hepatitis C in Europe from the patients' perspective: a survey in 5 countries. BMC Gastroenterol. 2013;13:16.

16. El Khoury AC, Vietri J, Prajapati $G$. The burden of untreated hepatitis $C$ virus infection: a US patients' perspective. Dig Dis Sci. 2012;57(11):2995-3003.

17. Liu GG, DiBonaventura M, Yuan Y, Wagner JS, L'Italien GJ, Langley P, et al. The burden of illness for patients with viral hepatitis C: evidence from a national survey in Japan. Value Health. 2012;15(1 Suppl):S65-71.

18. Younossi ZM, Tanaka A, Eguchi Y, Lim YS, Yu ML, Kawada N, et al. The impact of hepatitis C virus outside the liver: evidence from Asia. Liver Int. 2017;37(2):159-72.

19. Sun $\mathrm{S}$, Chen J, Johannesson $M$, Kind $P, X u L$, Zhang $Y$, et al. Population health status in China: EQ-5D results, by age, sex and socio-economic status, from the National Health Services Survey 2008. Qual Life Res. 2011;20(3):309-20

20. Afendy A, Kallman JB, Stepanova M, Younoszai Z, Aquino RD, Bianchi G, et al. Predictors of health-related quality of life in patients with chronic liver disease. Aliment Pharmacol Ther. 2009;30(5):469-76.

21. Deng $Q$, Wang $L M$, Zhang M. Quality of life and related influencing factors in Chinese adults. Zhonghua liu xing bing xue za zhi = Zhonghua liuxingbingxue zazhi. 2016;37(2):243-7.

22. Racciatti D, Gorgoretti V, Sepede G, Gambi F, Pizzigallo E. An Italian study on health-related quality of life and fatigue in patients with chronic fatigue syndrome and patients with chronic HCV virus infection: similarities and differences. Int J Immunopathol Pharmacol. 2011;24(3):673-81.

23. Kawaguchi Y, Mizuta T. Interaction between hepatitis $C$ virus and metabolic factors. World J Gastroenterol. 2014;20(11):2888-901.

24. Yamini D, Basseri B, Chee GM, Arakelyan A, Enayati P, Tran TT, et al. Tobacco and other factors have a negative impact on quality of life in hepatitis $C$ patients. J Viral Hepat. 2011;18(10):714-20.

25. Basseri B, Yamini D, Chee G, Enayati PD, Tran T, Poordad F. Comorbidities associated with the increasing burden of hepatitis $C$ infection. Liver Int. 2010;30(7):1012-8.

26. Sagnelli E, Coppola N, Pisaturo M, Masiello A, Tonziello G, Sagnelli C, et al. HBV superinfection in HCV chronic carriers: a disease that is frequently severe but associated with the eradication of HCV. Hepatology. 2009;49(4):1090-7.

27. Dai CY, Yu ML, Chuang WL, Lin ZY, Chen SC, Hsieh MY, et al. Influence of hepatitis $C$ virus on the profiles of patients with chronic hepatitis $B$ virus infection. J Gastroenterol Hepatol. 2001;16(6):636-40.

28. Gillis J, Cooper C, Rourke S, Rueda S, O'Brien K, Collins E, et al. Impact of hepatitis B and C co-infection on health-related quality of life in HIV positive individuals. Qual Life Res. 2013;22(7):1525-35.

29. Heeren M, Sojref F, Schuppner R, Worthmann H, Pflugrad H, Tryc AB, et al. Active at night, sleepy all day-sleep disturbances in patients with hepatitis C virus infection. J Hepatol. 2014;60(4):732-40.

30. Abdo AA. Hepatitis $C$ and poor quality of life: is it the virus or the patient? Saudi J Gastroenterol. 2008;14(3):109-13.
31. Amodio P, Salari L, Montagnese S, Schiff S, Neri D, Bianco T, et al. Hepatitis C virus infection and health-related quality of life. World I Gastroenterol. 2012;18(19):2295-9.

32. Lin $K$, Yang $X$, Yin G, Lin S. Diabetes self-care activities and health-related quality-of-life of individuals with type 1 diabetes mellitus in Shantou, China. J Int Med Res. 2016:44(1):147-56.

\section{Ready to submit your research? Choose BMC and benefit from:}

- fast, convenient online submission

- thorough peer review by experienced researchers in your field

- rapid publication on acceptance

- support for research data, including large and complex data types

- gold Open Access which fosters wider collaboration and increased citations

- maximum visibility for your research: over $100 \mathrm{M}$ website views per year

At BMC, research is always in progress.

Learn more biomedcentral.com/submissions 